\title{
Sur les pas des hommes qui ont vu l'ours. La conservation de la faune sauvage, un travail réel
}

\author{
Dorothée Denayer ${ }^{1}$, Catherine Mougenot $^{2}$, Damien Collard ${ }^{3}$ \\ 1 Biologie, sciences de l'environnement, Université de Liège, Département des Sciences et gestion de l'environnement, Unité \\ SEED, 6700 Arlon, Belgique \\ 2 Sociologie, Université de Liège, Département des Sciences et gestion de l'environnement, Unité SEED, 6700 Arlon, Belgique \\ 3 Sciences de gestion, Université de Franche-Comté, UFR STGI, Département AES/Droit, 90016 Belfort cedex, France
}

Si l'ours a fait l'objet de nombreux articles scientifiques, ce n'est pas le cas des humains qui œuvrent à sa conservation. L'objectif de cet article est d'approcher au plus près le travail des agents de la conservation. On y retrouvera une description fine et précise des diverses facettes de ce travail, que NSS a déjà explorées dans des livraisons précédentes (NSS 2003, 11, 4 ; NSS 2012,20,3), mais aussi une invitation originale à recourir à d'autres champs disciplinaires peu sollicités par les sciences de l'environnement, à savoir la psychodynamique du travail et l'ergologie. Une ouverture disciplinaire qui permet de rapprocher « souffrance » au travail et reconnaissance du « travail réel ».

La Rédaction

\section{Mots-clés :} conservation ; ours brun ; travail réel ; praticiens

Résumé - Le travail des acteurs de la conservation de la faune sauvage est un sujet peu abordé dans la littérature francophone. Prenant au sérieux les activités concrètes de ces acteurs, notre article propose un concept intermédiaire pour reconnaître les spécificités des métiers concernés. Ce concept repose sur l'identification et la description de quatre domaines d'activités - en synergie et en tension - qui sont au cœur de leur travail : "produire des connaissances", "prendre soin », "rendre compte » et "vivre avec ». L'analyse proposée reste proche des questions et des hésitations des professionnels, mais elle entend aussi monter en généralité et combler ainsi le "vide» si souvent décrit dans la littérature entre théorie et pratique. Notre démarche pragmatique est alimentée par l'exemple du projet de conservation de l'ours dans les Pyrénées françaises. Celui-ci est emblématique, même si jusqu'ici, il a surtout attiré l'attention par les nombreux conflits qui ont entouré sa mise en œuvre.

\section{Keywords:} conservation; brown bear; real work; fieldworkers

\begin{abstract}
Conservation of the brown bear: a multifaceted task. Bridging the analytical gap between theory and fieldwork in conservation. The Brown Bear Conservation Scheme in the French Pyrenees is highly emblematic. But the attention it has caused is due mainly to the many conflicts arising from its implementation. Our paper proposes to look deeper into this case in order to address an issue that has been greatly neglected: the work of wildlife conservation fieldworkers. The paper starts with a review of literature in this field and shows that the literature is essentially normative and does not consider the actual work of practitioners. Taking a closer look at their actual activities, our purpose is to develop an analytical framework aimed at filling the persistent gap between theory and practice often described in the literature. This framework is developed both empirically, as applied to the Brown Bear Conservation Scheme, and in a synthetic way, as an intermediary tool that could potentially apply to a wider range of cases. "Generating knowledge", "Taking care of », "Reporting " and "Living with »: these four aspects, which function in synergy and in tension, are the constitutive analytical elements of our proposal. Our paper aims to bring to the fore the specific issues involved in conservation work and if possible open the way to a connection with the work sciences (ergology, psychodynamics of work) that until now have paid very little attention to the management of wilderness and the environment.
\end{abstract}

Auteur correspondant : D. Denayer, d.denayer@ulg.ac.be 
«Si votre boulot, c'est de le suivre... pourquoi ne pouvez-vous pas nous dire où il est ? » La scène a lieu au printemps 2010 à Saint-Laurent-de-la-Cabrerisse, au pied des Pyrénées-Orientales. Un journaliste interpelle l'ingénieur responsable du suivi de l'ours brun dans les Pyrénées françaises. La veille, des traces ont été repérées par un chasseur sur le territoire de cette petite commune de l'Aude, bien loin du domaine vital du prédateur. Tous les indices donnent à penser qu'il s'agit de Balou, un ours slovène réintroduit dans le massif pyrénéen quatre années plus tôt. Mais aujourd'hui, où se trouve-til ? À ce stade, personne ne peut le dire avec certitude. Les agents de suivi de l'Office national de la chasse et de la faune sauvage (ONCFS) sont là pour tenter de le localiser. Pendant plusieurs heures, ils parcourent les Corbières à la recherche de l'animal, forts de leur connaissance fine de son comportement et des outils sophistiqués qu'ils utilisent. Sans succès: l'ours leur échappe. Les journalistes cherchent à comprendre pourquoi Balou est introuvable. Le suivi n'est-il pas performant? Les moyens mis en œuvre sont-ils insuffisants? Le silence des responsables du projet est-il le reflet d'un manque de transparence, d'une volonté de préserver la tranquillité de l'animal ? Les missions du « projet ours brun " sont officiellement scientifiques et techniques, tout comme l'est le suivi de l'ours dont les acteurs sont responsables. Mais le plan de conservation de l'espèce annonce aussi la nécessité de prendre en compte les "dimensions humaines » de ce projet régulièrement contesté : il faut sensibiliser le grand public et offrir des compensations aux acteurs locaux qui cohabitent avec le prédateur. Sur le terrain, les compétences réelles des acteurs du projet entremêlent volontiers différentes dimensions que l'on annonce pourtant comme "séparées ». Le récit d'une journée type de travail révèle les détails du suivi de l'ours, les met en lien avec l'histoire des réintroductions de spécimens slovènes dans les Pyrénées et pointe les difficultés de communiquer sur ces différents sujets. Plus que des dimensions, ce sont des séquences d'actions distinctes, mais totalement reliées, qui constituent les enjeux du travail de conservation de la faune sauvage. Le cas de l'ours pyrénéen a surtout attiré l'attention par les nombreux conflits qui l'entourent (Benhamou et Mermet, 2003). Au-delà des tensions liées à la présence d'un tel prédateur dans le massif, le plan de conservation fait lui-même l'objet de contestations. Certaines émanent de la communauté scientifique et portent notamment sur les enjeux génétiques des opérations de réintroduction. Dans cet article, nous revenons sur ce cas pour étudier la question du travail de conservation peu abordée par ailleurs. Notre projet vise à « équiper » ce dernier, autrement dit à proposer une conception générale de ses activités, qui restent toujours multiples et hétérogènes, afin de permettre leur comparaison et de travailler à leur reconnaissance. Prenant au sérieux leurs préoccupations, le cœur de notre propos consiste à analyser le travail de conservation en proposant un "concept intermédiaire » au sens de Becker (1998). Celui-ci entend monter en généralité sans morceler la réalité, c'est-à-dire en témoignant des enjeux spécifiques du métier, et en restant proche de ses questions et de ses hésitations.

Avant d'en arriver à la présentation de ce concept, nous nous sommes intéressés à la littérature qui aborde la question de plusieurs manières, mais sans se pencher véritablement sur les pratiques concrètes de la conservation. Lorsque celles-ci sont mises en avant par les praticiens, il semblerait que ces derniers peinent à les valoriser. En effet, soit ils tendent à les réduire aux objectifs généraux définis dans les plans d'action, et ce faisant les écrasent, soit ils mettent en avant les particularités du projet sur lequel ils travaillent, mais s'interdisent alors toute montée en généralité. Le concept intermédiaire que nous présentons vise à sortir de cette ornière. Nous le développons de manière empirique en l'appliquant au projet de conservation de l'ours. Pour finir, nous discutons notre proposition en soulignant l'intérêt d'appréhender la conservation comme un travail « réel ». Le travail « réel », ou l' « activité », est ce qui « est » réellement fait par les praticiens, il ne se résume pas au «travail prescrit », ou à la « tâche », c'est-à-dire ce qui « doit » être fait. Les ergonomes soulignent en effet que l'activité déborde nécessairement la tâche, dans quelque situation professionnelle que ce soit (Daniellou et al., 1983). Cette distinction est selon nous essentielle et nous conduit à proposer un rapprochement avec les sciences du travail jusqu'ici peu intéressées par la gestion de la nature et de l'environnement.

\section{De réflexions gestionnaires et pédagogiques à l'analyse du travail « réel »}

En rencontrant sur le terrain des praticiens de la conservation, nous faisons le pari qu'il est possible de construire sans a priori un tableau significatif de leurs activités (Sandberg et Tsoukas, 2011). Mais nous voulons aussi saisir la manière dont ce métier est exploré à travers les publications scientifiques, et cela en prenant en compte leurs thématiques, leurs concepts privilégiés et leurs publics cibles.

Deux revues scientifiques francophones sont consacrées aux interactions entre les sociétés, les natures et les environnements. En vingt années de parution, Natures Sciences Sociétés a inscrit le débat sur la recherche interdisciplinaire au cœur de sa problématique. En revanche, la revue n'a publié que deux articles traitant de manière explicite du travail des praticiens. Dans la seconde revue, Vertigo, nous n'en avons identifié aucun. Force est 
de constater qu'en matière de gestion de l'environnement et de conservation, si les projets en tant que tels occupent une part importante des réflexions, la question du travail ou du métier n'y est que peu ou pas traitée de manière directe.

Dans la littérature anglophone, en revanche, nous avons rapidement identifié plusieurs revues entièrement dédiées à la conservation de la faune et de la flore, le plus souvent ancrées dans les sciences naturelles, mais ouvertes aux contributions issues des sciences humaines. L'une d'elles, Conservation Biology, propose de nombreuses contributions partant d'un même constat: face aux résultats mitigés de la conservation, il faut concevoir des actions plus performantes, en réduisant l'écart persistant entre les recommandations scientifiques et les résultats obtenus sur le terrain. De nombreux auteurs imputent cet écart au manque de maîtrise des dimensions humaines des projets. Trois types de réflexions alimentent ce débat.

Le premier type propose de redéfinir les objectifs de la conservation pour mieux guider les acteurs de terrain (Mascia et al., 2003 ; Reyers et al., 2010). Comme la conviction des biologistes est que les connaissances scientifiques au sujet des espèces à protéger trouvent forcément une utilité directe, l'efficacité toujours partielle des projets doit s'expliquer autrement. Des missions nouvelles seraient à définir avec l'appui des sciences sociales. Dans cette perspective, la prise en compte de la diversité des intérêts du public face à la conservation de la vie sauvage (Teel et Manfredo, 2010), le choix d'un apprentissage social et l'engagement dans une gestion adaptative (Cundill et al., 2011) sont autant d'objectifs qui devraient désormais faire partie des politiques et se décliner dans les plans de gestion.

De manière parallèle ou parfois entremêlée, la deuxième proposition est de revoir les cursus académiques des praticiens, biologistes en majorité (Orr, 1999). Ici, le problème est défini comme une défaillance des universités, qui échouent à former efficacement des acteurs de la conservation. Enseignants et professionnels sont appelés à s'exprimer sur les nouveaux cursus à promouvoir (Muir et Schwartz, 2009). La nécessité d'un bagage théorique fort est réaffirmée, mais son contenu doit s'ouvrir à des disciplines autres que les sciences naturelles (Brewer, 2006). Il importe en effet de doter les candidats à ces métiers de compétences humaines pour éviter d'en faire des « savants idiots » (Jacobson et Duff, 1998). Les auteurs soulignent les efforts consentis pour accorder une place plus importante aux travaux de terrain comme source d'apprentissage. Et parmi les nouvelles aptitudes invoquées, les suivantes émergent : " savoir travailler en groupe ", "savoir dialoguer ", ou encore « être prêt à s'adapter » (Cannon et al., 1996). Ces propositions portent à la fois sur les contenus et sur les modes d'apprentissage et elles doivent s'appliquer dans le cadre d'un cursus académique. Elles doivent être définies de manière explicite, rester en nombre limité et toujours préexister à la mise en œuvre des travaux de terrain.

Un troisième axe de réflexion attire enfin l'attention sur le travail quotidien des professionnels de la conservation, en soulignant que ces acteurs constituent en réalité des groupes extrêmement hétérogènes et dont les compétences sont particulières (Muir et Schwartz, op. cit.). Ils déplorent eux-mêmes le fait que leurs expériences soient si difficilement valorisables et leur reconnaissance si faible au sein des mondes institutionnels (Noss, 1997 ; Cundill et al., op. cit). Compte tenu du caractère non académique et peu reconnu des activités mises en œuvre sur le terrain, les auteurs font état des inquiétudes des chercheurs et des enseignants (Blickley et al., 2013). Cette troisième approche, de loin la plus discrète, se résume souvent à l'identification d'une défaillance: le fameux "gap between theory and practice».

Entre une réflexion sur le travail prescrit (dans les programmes), les savoirs à dispenser (dans les cursus) et cette dernière réflexion qui porte sur le travail « réel », il reste un pas difficile à franchir, voire même à identifier. Deux raisons non exclusives peuvent selon nous expliquer ce blocage. D'abord le fait que les recherches en sciences sociales se focalisent prioritairement sur les conflits ou les oppositions entre groupes d'acteurs qui caractérisent fréquemment ces projets. On étudie alors les intérêts, savoirs et représentations des différents groupes en présence, en renforçant l'idée que les problèmes ne relèvent pas du projet lui-même, mais de ses interactions avec le reste du monde. Ensuite les (études) scientifiques témoignent visiblement d'une certaine réticence à adopter un regard réflexif, à admettre que les praticiens de la conservation ne pratiquent pas la "même science » que les chercheurs de laboratoire et qu'ils ne peuvent y appliquer des critères de validation standardisés. Ce fait est acceptable s'il est rapporté à la complexité et aux singularités d'un terrain toujours en partie imprévisible. En revanche, s'il caractérise le travail des professionnels, il pose problème. Les articles font alors mention d'un « vide ", toujours à combler. Or nous pensons que cet écart - entre la conservation telle qu'elle est planifiée et ce qui est mis en œuvre sur le terrain, en termes de pratiques, de connaissances, de résultats - n'est pas un vide contre-productif, mais plutôt un " plein » : c'est la réalité du travail des acteurs de la conservation, qui déborde de bien des manières du cadre technicoscientifique. Une réalité que l'on ne peut anticiper, difficile à saisir, car encombrée d'expériences, d'apprentissages, d'hésitations et de manières de faire, de détails et d'anecdotes qui constituent la part concrète du travail. Et c'est précisément ce plein, mal défini, que nous souhaitons analyser ici.

Notre enquête a porté sur une dizaine de projets de conservation de la faune dans différents pays au nord et 


\section{La conservation, un travail prescrit}

\section{Volet « nature »}

Étape 1 : État des lieux

sur la base d'indicateurs

de conservation

Étape 2 : Identification

des menaces et mesures

d'action (protection,

restauration, etc.)

Étape 3 : Mise en œuvre des

décisions de gestion de la

nature

Étape 4 : Monitoring et suivi

de l'évolution

Étape 5 : Rapports d'activités et publications scientifiques

\section{$+$}

\section{Volet « humain »}

Étape 1 : Sensibilisation

et information du public

Étape 2 : Concertation

Étape 3 : Propositions

de mesures d'accompagnement

et de compensation
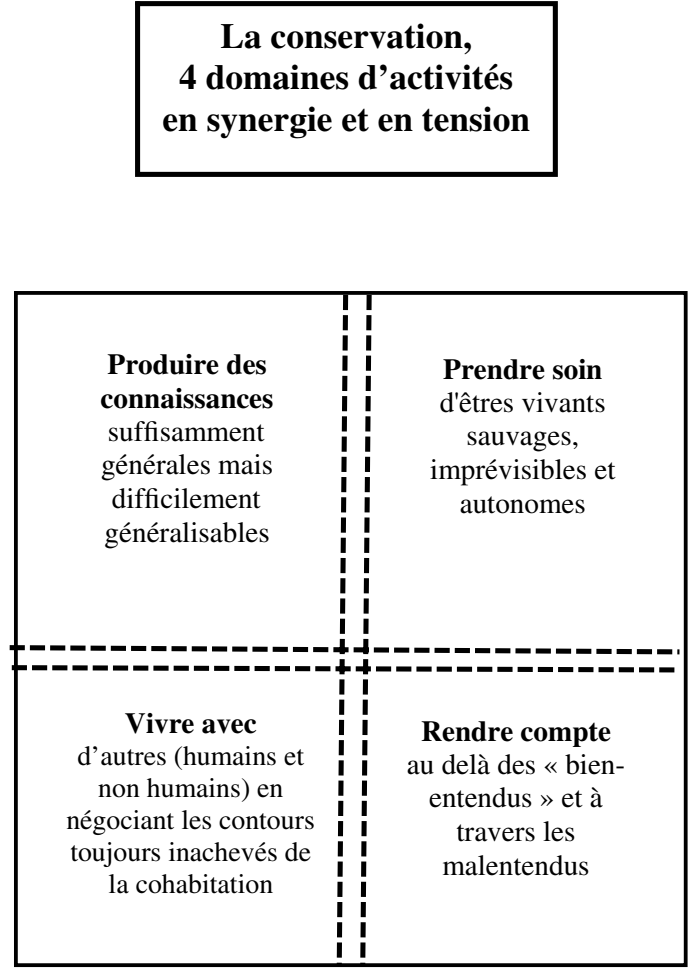

La conservation, un travail réel ....après des heures d'une marche intense, rester attentif au moindre indice; une touffe de poil, une fourmilière éventrée : trouver une trace et l'examiner; plus loin, en trouver une autre ; interpréter leur signification : les indices se recoupent, se complètent les uns les autres, constituant des faisceaux de renseignements ; entrer dans le monde de l'animal : il a fait ceci, est passé par là, peut-être est-il tout près ; pendant quelques minutes, s'imaginer ce que serait la confrontation; sortir de cette rencontre pour rentrer au bureau; encoder les informations dans la base de données ; recevoir une information d'un autre ordre : un troupeau de chèvres a été attaqué ; se rendre sur les lieux, avec appréhension ; remonter à pied les flancs du vallon au côté de l'éleveur ; examiner les lambeaux de chairs et d'os ; achever les animaux agonisants ; dresser un constat, rédiger un rapport ; tenter d'apaiser l'éleveur ; prendre quelques minutes pour digérer la confrontation ; retourner terminer l'encodage des données : le rapport d'activités doit être préparé...

Fig. 1. Un concept intermédiaire sous la forme de quatre domaines d'activités en synergie.

Une proposition pour équiper le passage de la conservation prescrite à la conservation dans le monde réel, explorer le travail réel des acteurs de la conservation dans toutes les tensions qui le traversent et ainsi contribuer à sa reconnaissance.

au sud. Concrètement, nous avons mené une série d'entretiens compréhensifs auprès de praticiens. Afin de saisir en quoi consiste leur travail, nous avons procédé à l'observation directe de leurs activités (Denayer, 2013). Les traces écrites de leurs pratiques (rapports, publications, autobiographies) ont aussi permis de compléter ces données dans leur profondeur historique. Elles montrent des relations qui les engagent de manière déterminante, sans forcément être visibles dans l'instantané des situations de travail. En suivant ces professionnels, nous avons alors cherché à cerner les contours des défis concrets qu'ils ont à relever. "Produire des connaissances», «prendre soin» (des animaux sauvages), « rendre compte» et «vivre avec »: ce sont là quatre domaines d'activités que nous identifions comme partagés par tous et qui dépassent ou reconfigurent autrement les missions définies pour chaque projet. L'analyse que nous proposons est ici appliquée à l'une de nos principales études de cas, le projet de conservation de l'ours brun dans les Pyrénées françaises, que nous présentons également de manière synthétique dans la figure 1 . Nous montrons comment cette étude permet d'explorer les défis relevés par les acteurs de ce projet particulier, tout en les rattachant aux tensions plus générales qui traver- sent l'ensemble des projets de conservation de la faune sauvage. Des tensions inhérentes à ces métiers. En effet, pour les acteurs que nous avons rencontrés, travailler signifie « faire avec » ces tensions, les affronter, mais non les supprimer.

\section{Les quatre domaines d'activités de la conservation}

\section{Produire des connaissances}

« Notre boulot? C'est le suivi technique et scientifique des ours bruns dans les Pyrénées ». Les missions officielles des membres de l'équipe technique ours et du réseau ours brun de l'ONCFS paraissent claires : organiser, mettre en œuvre le suivi de l'ours brun, centraliser et analyser les données qui en sont issues. Les objectifs de ce monitoring sont multiples : évaluation de l'état de santé de la population, localisation des sites régulièrement fréquentés, analyse des déplacements des individus, etc. Ces missions se déclinent en deux formes d'organisation collective et de production de connaissances, qui constituent donc deux types de suivi différents, mais 
néanmoins complémentaires et à articuler : 1'un est dit « patrimonial », l'autre « télémétrique».

Le premier existe depuis plusieurs décennies. Il est « indirect » car basé sur la récolte d'indices de présence : empreintes de pattes, poils, pistes, déjections ou griffures à même les arbres. Il implique de longues heures de déambulation dans la montagne, une connaissance fine du milieu, un coup d'œil indéniable, et de grandes qualités physiques. Si ce mode de suivi s'étale sur toute l'année, les indices sont rares. L'ours n'est finalement que très peu aperçu et l'un des enjeux de l'entreprise réside dans l'interprétation de son comportement : il est passé par ici, il devrait probablement repasser par là... Le suivi patrimonial est pratiqué par une diversité d'acteurs (près de 200 individus!) réunis au sein du réseau ours brun. Leur principal point commun est leur connaissance du territoire et leur attachement à celui-ci, et notamment aux richesses naturelles qu'il abrite.

Le suivi télémétrique est, quant à lui, directement lié aux réintroductions d'ours slovènes dans les Pyrénées. $C^{\prime}$ est en effet à l'occasion de la capture puis du lâcher des animaux en France que ces derniers ont été équipés de manière à émettre des signaux permettant de les localiser. L'antenne réceptrice et l'ordinateur compilant les données GPS deviennent des outils incontournables pour obtenir et traiter une importante quantité d'informations. L'ours, animal si discret, peut dès lors être suivi de beaucoup plus près et ces connaissances rapprochées impliquent de nouvelles responsabilités : il devient théoriquement possible d'anticiper les attaques des troupeaux ou d'indiquer aux fédérations de chasseurs les zones à éviter. Mais les émetteurs ont une durée de vie limitée à celle de leur batterie et bientôt, les animaux ainsi médiatisés redeviennent des ours comme les autres : furtifs et inaccessibles, si ce n'est par l'intermédiaire des traces, poils et empreintes qu'ils laissent derrière eux. Le suivi télémétrique est pratiqué par un collectif restreint : les agents du «pôle suivi » de l'équipe technique ours de l'ONCFS.

Dans un cas comme dans l'autre, les acteurs du suivi ont toujours un temps de retard sur l'ours. Pourtant, officiellement, outre l'amélioration des connaissances sur la population, le projet affiche un objectif secondaire opérationnel : la maîtrise, le contrôle des individus et en particulier des animaux relâchés. "Si cela ne se passait pas comme prévu, nous avions mis dans le plan que nous pourrions recapturer les ours, les retirer du milieu ».

Le «projet ours brun » dans les Pyrénées françaises n'est pas un cas isolé. Partout dans le monde, la conservation de la faune menacée se veut fondée sur la connaissance des dynamiques naturelles. Ceci passe généralement par la réalisation d'un état des lieux et un suivi. Mais les animaux ne cessent de se dérober aux explorations des praticiens. Combien sont-ils ? Où sont-ils ? Comment vont-ils? Sur le terrain, produire des connaissances ancrées dans des situations concrètes est un enjeu qui comporte ses propres défis. Ces connaissances restent toujours provisoires et supposent la participation régulière de coproducteurs venus d'horizons variés : chasseurs, pêcheurs, citoyens motivés et volontaires. Par ailleurs, si le projet est contingent aux conditions naturelles du terrain, il est aussi perméable à son environnement humain (social, historique, citoyen). Pour des biologistes plongés dans l'action, la diversité des connaissances à produire afin de mener à bien toutes leurs missions est surprenante : techniques et scientifiques, mais aussi largement ouvertes et pragmatiques. Connaître les acteurs locaux, leurs projets et leurs points de vue est une condition essentielle pour les comprendre, les informer et répondre à leurs inquiétudes ou faire fonctionner un réseau d'observateurs tel que le réseau ours brun. À cette liste de savoirs nécessairement situés s'ajoutent ceux relatifs à l'histoire, à la politique, à l'administration, aux rapports humains, à la diplomatie, ou encore aux médias. Les membres du projet accumulent des connaissances " générales » qui restent difficilement valorisables à travers les canaux reconnus de la conservation, mais qui améliorent - voire conditionnent - la qualité des interactions qu'ils doivent impérativement entretenir avec une multitude d'autres acteurs (partisans, opposants, monde politique, médias, etc.). Le suivi «scientifique et technique » des ours bruns implique la compilation de connaissances précises et scientifiques, mais toujours inscrites, reliées, dépendantes de connaissances très générales, et cette facette du travail des praticiens n'est pas annoncée dans le plan qui définit leurs tâches.

\section{Prendre soin}

Quand et comment intervenir? L'animal sauvage dont on prend soin est-il toujours sauvage ? La plupart des projets de conservation de la nature doivent composer avec ce paradoxe. D'un côté, la science répartit les organismes en groupes homogènes que sont les espèces, elle dépeint leur écologie comme une dynamique générale et relativement stable, de manière à pouvoir fixer des objectifs à atteindre en termes de conservation ou de restauration. Mais sur le terrain, les acteurs de la conservation ont affaire à des individus particuliers et sont de ce fait amenés à réviser régulièrement leur jugement sur ce dont les êtres dont ils s'estiment responsables sont capables ou pas. Revenons au cas de l'ours brun pour éclairer ce paradoxe. Au début des années 2000, c'est par le biais d'un partenariat inattendu entre le monde naturaliste et les autorités de quelques communes des Pyrénées centrales que naît le projet de réintroduction d'individus capturés en Slovénie. L'objectif est de restaurer en partie une population autochtone moribonde: subsistent alors quelques ours de souche pyrénéenne et, parmi eux, une seule et unique femelle. 
Le choix du pays source fait débat. Pour les généticiens, il est important que les individus réintroduits soient aussi proches que possible de la souche à restaurer. De ce point de vue, les candidats idéaux sont les spécimens espagnols. Mais pour des raisons politiques liées à la protection de ces derniers, un tel projet n'est pas réalisable. Les promoteurs de la réintroduction se tournent alors vers des partenaires slovènes. Et d'un point de vue purement écologique, l'acclimatation des ours slovènes est aujourd'hui considérée comme une réussite : ils se sont déplacés des Pyrénées centrales aux PyrénéesAtlantiques, se sont intégrés dans le milieu et se sont reproduits avec les spécimens autochtones. Bien entendu, l'ours en Pyrénées n'est pas sauvé pour autant. Huit individus réintroduits, $c^{\prime}$ est finalement très peu. $D^{\prime}$ autant que certains ont ensuite connu un destin tragique.

C'est bien peu et ce n'est pourtant pas rien! Car il s'agit d'abord de piéger l'animal à l'aide d'appâts ou à l'affût, dans des sites connus pour être régulièrement fréquentés. Lorsque l'occasion se présente, la cible est anesthésiée directement. Plus fréquemment, l'ours est piégé au moyen d'un lacet à patte relié à un dispositif d'alarme. Il faut dans ce cas réagir rapidement, dans l'heure qui suit le déclenchement du signal, pour éviter que l'animal ne se blesse en tentant de se libérer. Il est alors capturé et endormi et fait ensuite l'objet d'une liste impressionnante de manipulations : des examens généraux (taille, poids, sexe) et divers prélèvements (échantillons de sang, de peau, de poils, d'une dent - qui servira à déterminer son âge - et d'excréments). L'ours est également marqué de multiples manières : tatouage, marques auriculaires de couleur, puce électronique auriculaire, collier émetteur et, enfin, émetteur intraabdominal. Toutes ces actions engendrent inévitablement une série de soins vétérinaires. Commence alors le transport vers la France. L'animal est placé dans une cage (cylindrique, pour éviter toute blessure), installé à l'arrière d'une camionnette. Une caméra permet de surveiller son comportement durant tout le trajet et son réveil. Une fois arrivé sur le site prévu pour son relâcher, les portes sont ouvertes, laissant l'ours se précipiter vers son nouvel environnement.

Les acteurs rencontrés dans le cadre de notre enquête évoquent l'enthousiasme d'avoir vu de près et touché de tels animaux. Ils soulignent le caractère " hors du commun » de l'entreprise, se disent " privilégiés ». Ils témoignent également de l'attachement qu'ils développent pour ceux qui deviennent alors leurs "protégés ".

La rationalité technicoscientifique se double d'une approche sensible. La première envisage les animaux comme les représentants d'une espèce dont on peut anticiper la biologie et les besoins. Elle s'appuie sur des connaissances générales et mobilise des protocoles standards d'intervention. Mais au-delà de l'espèce et de ses exigences, les acteurs se préoccupent d'individus inventifs, poursuivant leur propre destin, parfois récalcitrants et toujours imprévisibles. En cela, la conservation de la faune entretient des rapports étroits avec le care tel qu'il est décrit par les sciences médicales et infirmières : prendre soin n'est pas faire des soins. Le soin ne peut être résumé à des actes purement techniques et les acteurs eux-mêmes s'en trouvent transformés : la relation qui se noue avec le « soigné » modifie leur rapport aux autres et à leurs pratiques, change leur vision du monde et soulève des questions éthiques. Celles qui se posent à tous ceux qui prennent soin du vivant, sous toutes ses formes. De ce triptyque qu'implique le soin - rationalité technicoscientifique, questionnement éthique et attachement sensible -, seul le premier volet est ouvertement affiché dans les plans de conservation et rapports officiels. Le deuxième est moins présent ou facultatif. Quant au troisième, il semble être considéré avant tout comme le témoignage d'un manque de maîtrise et d'objectivité, voire comme un signe de faiblesse.

\section{Rendre compte}

Nul professionnel n'est dispensé de rendre compte de son travail. S'agissant de la réintroduction de prédateurs tels que l'ours brun, aussi expérimentale et imprévisible que soit l'opération, il faut communiquer. Les praticiens de la conservation doivent traduire leur travail dans des formats variés, de façon formelle et souvent aussi informelle. Leurs publications scientifiques et leurs rapports sont le gage du sérieux et de l'aboutissement du projet qui leur a été confié. L'information et la sensibilisation sont toujours au menu de leurs tâches journalières, dans un contexte humain et social parfois tendu. Et de manière générale, la communication est désignée par les acteurs comme un enjeu fondamental pour la réussite et la pérennité de leur projet. Pour autant, ils se montrent en réalité plutôt sélectifs sur les éléments à propos desquels ils estiment pouvoir communiquer, notamment sur les opérations de relâcher et les données de localisation des ours. Que faut-il dire ? À qui ? À quel moment ? Pour gagner quoi ? Et en assumant quels risques ? Il s'agit de montrer de diverses manières que le travail de suivi porte ses fruits, que les ours sont localisés régulièrement, que leurs déambulations sont surveillées. Il n'est pour autant pas question de désigner la localisation exacte des tanières et autres sites de fréquentation régulière, au risque de voir certains opposants ou simples curieux s'en approcher et les endommager, ou, pire, s'en prendre aux animaux protégés.

De manière générale, il y a des informations que l'on partage et d'autres pas. La transparence ne peut être ni entière, ni sans bornes. Cela est vrai quel que soit le format privilégié pour communiquer. Les articles scientifiques effacent en particulier le caractère singulier de la production de connaissances, à savoir les relations aux humains et aux animaux dans lesquelles elle prend place. Les 
messages de sensibilisation tendent à simplifier fortement la réalité, sous prétexte qu'elle ne peut ou ne veut être entendue. Dans un cas comme dans l'autre, dire c'est forcément traduire et trahir, c'est enrichir la réalité et/ou l'appauvrir, et ce peu importe les ambitions d'objectivité des acteurs. C'est une réalité qui peut être difficile à assumer ouvertement, surtout pour des scientifiques. Chaque compte rendu doit contenir des messages spécifiques et s'adresser à un public ciblé, en espérant des retombées positives pour le projet. Mais il est également le point de départ de quelque chose d'inattendu : une fois délivré, il est saisi par des acteurs - sympathisants ou opposants qui utilisent son contenu pour leur propre compte. Alors, si la communication construit la confiance et la légitimité, elle est aussi susceptible d'exposer les praticiens à de nombreux malentendus. D'autant qu'il leur est toujours difficile d'éviter la contradiction entre des messages qui restent toujours partiels.

\section{Vivre avec}

Même si les acteurs du " projet ours brun » placent le suivi au cœur de leurs missions, ce programme est avant tout connu en raison de la controverse qui l'entoure et qui a accompagné les réintroductions. Un conflit en particulier apparaît en surbrillance : celui qui oppose les défenseurs de l'ours à certains éleveurs. Dans le monde de l'élevage pyrénéen, cet animal est régulièrement présenté comme une nouvelle contrainte insupportable et le projet de le conserver comme illégitime. Il faut dire que l'espèce, longtemps classée «nuisible » a fait jusqu'au milieu du $X X^{\mathrm{e}}$ siècle l'objet $\mathrm{d}^{\prime}$ une destruction systématique encouragée par l'administration. Alors si aujourd'hui l'espèce est protégée, les dégâts infligés par les ours aux animaux d'élevage sont très mal tolérés. Pour améliorer la cohabitation, de nombreuses mesures sont conçues et mises en œuvre. Et les éleveurs sont indemnisés lorsque les dégâts sont avérés. Mais les réintroductions ont donné une autre dimension à ce conflit. Peu de temps avant qu'elles n'aient lieu, l'ours brun était déclaré éteint dans les Pyrénées centrales. Quelques individus survivaient dans les Pyrénées-Atlantiques, là où en parallèle des bergers continuaient d'exercer leur activité. Mais, dans les Pyrénées centrales, plus personne n'avait à vivre avec cet animal. Alors, parmi les initiatives de cohabitation pour accompagner les réintroductions, on peut citer l'engagement de plusieurs bergers itinérants au sein du «pôle pastoral » de l'équipe technique ours. Équipés de l'outil télémétrique tant que cela est possible, ils sont chargés de repérer les zones d'élevage sensibles et d'y intervenir en soutien aux éleveurs, qu'ils soient partisans ou opposants. Dans cet effort, les praticiens de la conservation doivent travailler avec des partenaires divers : qui sont leurs alliés et comment en rallier de nouveaux ? Dans ce cas encore, ils ne peuvent répondre une fois pour toutes : au fil de l'histoire, dans le cours de l'action, la conduite de chacun peut rester incertaine. Un tel constat concerne aussi les animaux, qui sont les premiers à ne pas faire ce que l'on attend d'eux et à mettre en péril par leur comportement des compromis durement acquis. Les conflits ne sont pas rares, même lorsqu'un projet semble porter ses fruits.

Loin des principes abstraits du «win-win» et du «bien commun », construire un «vivre avec » n'est pas la mise en œuvre d'un équilibre harmonieux. Ainsi, la définition d'un protocole officiel "ours à problèmes » (sorte de garantie et de rempart « objectif » au cas où un individu «atypique» se montrerait particulièrement vorace et destructeur) se révèle beaucoup plus compliquée qu'il n'y paraît. À partir de combien de brebis dévorées la situation devient-elle problématique? Dès la première pour certains, tandis que pour d'autres cette question est balayée à grands renforts d'indemnisations financières. Qu'est-ce qu'un individu « atypique » ? Les spécialistes du comportement ursin affirment que l'ours passe au cours de sa vie par plusieurs phases associées à des comportements plus ou moins agressifs et téméraires. On peut citer le cas de Franska, femelle slovène relâchée dans les Pyrénées au printemps 2006. L'ourse a fait de nombreux dégâts, suscitant l'indignation et la colère des éleveurs. Ces derniers ont exigé qu'elle soit recapturée, extraite du milieu, au nom du protocole "ours à problèmes ». Ce ne sera pourtant pas fait et d'après le responsable du projet : «Elle ne rentrait pas dans la grille. Et pourtant, elle nous a effectivement posé d'énormes problèmes. Les gens ont commencé à faire des battues ». Il n'y aura pas d'accord sur le cas de Franska, celle-ci ayant finalement réglé la question à sa façon, plus intrépide que tout autre : sa course s'est brutalement interrompue sous les roues d'un véhicule au cours de l'été 2007. L'autopsie a établi que l'ourse slovène avait atteint l'âge de 17 ans - dix ans de plus que ce qu'avaient annoncé ceux qui l'avaient capturée en Slovénie. Cet âge avancé expliquerait au moins en partie un comportement jugé destructeur.

Le projet chemine selon une trajectoire collective, marquée d'évènements particuliers et toujours chargée de tensions. S'ils s'efforcent d'infléchir cette dernière pour atteindre leurs objectifs, les acteurs de la conservation sont pris dans un processus qu'ils ne maîtrisent jamais totalement. Les ours dont ils sont les porte-parole, les bergers dont ils sont les alliés, les éleveurs qui s'opposent régulièrement à eux... tous peuvent se montrer infidèles. D'accords en désaccords, de succès en défaites, chacun garde en mémoire les faits marquants. Bien plus qu'une chronologie de faits, loin d'une norme visant l'équilibre harmonieux et l'évacuation des conflits, le « vivre avec » construit une réserve d'histoires où puiser les raisons de s'estimer tantôt gagnant, tantôt perdant, mais le plus souvent les deux à la fois. 


\section{Discussion : la conservation, un travail « réel »}

En pointant le caractère déterminant et spécifique de l'engagement des acteurs dans l'accomplissement de leurs tâches, nous nous situons logiquement dans un continuum avec la troisième perspective - l'approche praticienne - que nous avons identifiée dans la littérature. Et nous rejoignons les auteurs qui mettent en évidence que l'engagement pratique des acteurs de la conservation n'est que peu, voire pas reconnu par les institutions académiques (Cundill et al., 2011). Mais notre proposition dépasse ce raisonnement. Car souligner le caractère concret et contingent des pratiques ne suffit pas. Pour être effective, leur reconnaissance suppose un compte rendu précis de ce qui est engagé dans les situations. L'« impératif scientifique » qui entoure la conservation, la volonté de l'envisager comme la mise en œuvre prévisible d'une science "pure», n'a jusqu'ici laissé que très peu de place à une approche inductive et pragmatique de l'aspect concret du travail des acteurs. Pratiquer un tel métier suppose de définir des objectifs qui doivent nécessairement être débattus sur le terrain et d'apprendre à tirer parti des situations. Ce métier s'ancre dans des pratiques qui ne peuvent se résumer à leurs dimensions scientifiques, qu'elles soient naturelles ou humaines. Il implique un détour riche d'enseignement sur les manières de concevoir les plans et les formations des acteurs de la conservation. Et c'est bien cette direction qu'ont voulu aussi explorer Granjou et Mauz (2012) dans un des deux articles francophones évoqués plus haut. Ce sont les tensions du travail « réel » des « acteurs entrepreneurs » qui œuvrent au rapprochement de l'agriculture et de la conservation de la nature.

Notre analyse propose des prises pour mieux saisir les projets et les comparer entre eux. Les questions qu'elle invite à poser laissent une place importante aux verbes d'action, sans préjuger de l'identité de ceux qui y participent. Produire des connaissances en tout genre et les mettre en ordre. Les diffuser le plus largement possible ou plutôt les garder discrètes. Redéfinir ce que la nature devient autant que lâcher prise et accompagner. Faire avec des partenaires imprévisibles, des animaux mais aussi d'autres humains. Imaginer l'avenir avec eux en gardant la mémoire des faits passés. Puis, finalement, décider de manière toujours provisoire. Tous ces enjeux doivent être pris comme un ensemble, indissociables, dans une dynamique commune qui les voit déborder les uns sur les autres. Les domaines d'activités que nous proposons ne sont pas des territoires ou des champs bornés une fois pour toutes. Ils sont au contraire amenés à se croiser et à se prolonger dans le temps. Il est ainsi crucial de comprendre le suivi de l'ours comme un projet de connaissance qui contribue à la construction d'un vivre ensemble sur un territoire. Toute opération de sensibilisation ou de cohabitation est inévitablement impactée par les choix qui sont opérés dans le cadre du projet, de manière apparemment «purement » technique. C'est le cas lorsque naît l'idée d'utiliser l'instrument télémétrique pour équiper les bergers itinérants. Ces actions concourent simultanément à plusieurs objectifs. En les suivant, on découvre les confrontations des praticiens de la conservation avec un monde qui résiste à leurs projets, guide leurs apprentissages du terrain autant que la construction de leur expertise. Des professionnels qui doivent ruser en permanence face à ce «réel » qui leur échappe. Face à des problèmes très concrets, loin des objectifs officiels souvent lisses et abstraits, la réponse qu'ils cherchent est éminemment pratique. Alors, ils y engagent leur formation scientifique, mais aussi leur sensibilité, leur capacité à porter et à supporter le projet. Ils s'inscrivent dans «des histoires » et travaillent en construisant des compromis toujours provisoires.

Ces négociations reposent sur des articulations continuelles, mais le plus souvent invisibles (Star et Strauss, 1999), voire clandestines. Car pour bien travailler, il faut pouvoir s'éloigner des recommandations et des règlements. Les quatre domaines d'activités de la conservation que nous mettons en avant échappent $d$ 'ailleurs très largement aux indicateurs qui permettent de piloter les projets de conservation et d'attester de leur bien-fondé scientifique. Notre analyse invite à prêter attention au fait que les résistances qui pèsent sur ces projets ne viennent pas seulement de l'extérieur. Autrement dit, elles $n^{\prime}$ ont pas leur source uniquement dans les conflits entre les partenaires. Les tensions résultent aussi de ce que ces missions sont définies sur la base de postulats qui portent en eux des contradictions. Ainsi, le principe selon lequel les espèces sauvages retrouvent leur autonomie dans la restauration d'un état de référence idéalisé doit composer avec une nature de fait manipulée et toujours «en devenir». Cet autre principe qui n'admet la connaissance que comme une somme de vérités générales, objectives et intemporelles se confronte à la nécessité d'appréhender des situations dans tous les détails spécifiques qui font de chaque problème un cas particulier. Pour les acteurs de la conservation, se pose sans cesse la question de savoir comment il est possible de prendre parti à la fois pour ces « habitants-là » et ces " animaux sauvages-là », alors que tous restent imprévisibles et poursuivent leurs propres projets. Et si un principe de transparence postule que la confiance s'acquiert par le biais d'une communication intense, dans les faits, chaque compte rendu est une traduction susceptible d'être interprétée tantôt comme une expertise, tantôt comme une trahison. Et il expose les acteurs aux soupçons et aux malentendus.

Les quatre domaines d'activités que nous proposons sont spécifiques, ils comportent leurs propres enjeux et ne fonctionnent pas sur le même mode. Concrètement, leur complémentarité n'est pas déterminée et leurs manières de se chevaucher doivent sans cesse être renégociées. 
Indissociables, ils sont tout à la fois en tension et en synergie. Nous découvrons chemin faisant que nos propres réflexions ont de très nombreux points communs avec deux approches qui trouvent leur origine dans l'analyse du travail industriel, à savoir la psychodynamique du travail et l'ergologie. Celles-ci revendiquent un même point de départ : l'écart entre le travail prescrit et le travail « réel », mis en évidence par les ergonomes dans les années 1970. Ces deux perspectives, illustrées par les travaux de Dejours $(2001$; 2013) et de Schwartz $(2007 ; 2010)$, signent ainsi une critique décisive adressée au taylorisme, cette théorie scientifique du travail qui suppose que la meilleure manière de produire peut être décrite de façon objective. Ces approches s'alimentent d'une richesse conceptuelle commune : l'écart entre prescription et situation permet et suppose un engagement vital au travail, une intelligence collective et le déploiement de ruses. Le travail « réel » traverse et mobilise le corps autant que l'esprit. Et, comme le souligne Dejours (2001) : «Le réel se fait connaître par sa résistance aux procédures, aux savoir-faire, à la technique, à la connaissance, c' est-à-dire par la mise en échec de la maitrise ». Si le manque de reconnaissance du travail « réel » peut être une source de souffrance pour les acteurs de terrain, nous avons aussi constaté que leur attachement à la science, et à la maîtrise qu'elle suppose, les amène souvent à douter de la pertinence de leurs actions. Presque invisible, le travail « réel » se dit peu ou mal. D'où la difficulté de l'évaluer et même d'en rendre compte. C'est alors une posture modeste qui est requise pour les chercheurs qui prétendent s'en saisir et l'analyser. La psychodynamique du travail et l'ergologie, les sciences du travail en général, se sont déployées sur de nouveaux et nombreux terrains, bien au-delà de la question du travail industriel. $Q u^{\prime}$ ont-elles à nous apprendre sur les métiers dédiés à la gestion de la nature et de l'environnement? Cette question ouvre selon nous de nouveaux chantiers, de nouvelles perspectives interdisciplinaires qui, en se focalisant sur l'activité, refusent d'adresser aux mondes naturels et sociaux des prescriptions séparées.

\section{Références}

Becker H.S., 1998. Les ficelles du métier. Comment conduire sa recherche en sciences sociales, Paris, La Découverte.

Benhamou F., Mermet L., 2003. Stratégie et géopolitique de l'opposition à la conservation de la nature : le cas de l'ours des Pyrénées, Natures Sciences Sociétés, 11, 4, 381-393.

Blickley J.L., Deiner K., Garbah K., Lacher I., Meek M.H., Prensky L.M., Wilkerson M., Winford E.M., Schwartz
M.W., 2013. Graduate student's guide to necessary skills for nonacademic conservation careers, Conservation Biology, 27, 1, 24-34.

Brewer C., 2006. Translating data into meaning: education in conservation biology, Conservation Biology, 20, 3, 689-691.

Cannon J.R., Dietz J.M., Dietz L.A., 1996. Training conservation biologists in human interaction skills, Conservation Biology, $10,4,1277-1282$

Cundill G., Cumming G.S., Biggs D., Fabricius C., 2011. Soft systems thinking and social learning for adaptive management, Conservation Biology, 26, 1, 13-20.

Daniellou F., Laville A., Teigner C., 1983. Fiction et réalité du travail ouvrier. Les cahiers français, 209, 33-45.

Dejours C., 2001. Subjectivité, travail et action, La pensée, 328, 719.

Dejours C., 2013. Travail vivant (1): sexualité et travail, Paris, Payot et Rivages.

Denayer D., 2013. Produire des connaissances, prendre soin, vivre avec et rendre compte. Une analyse comparée des compétences des acteurs de la conservation de la faune. Thèse de doctorat, Liège, Université de Liège.

Granjou C., Mauz I., 2012. Des espaces frontières d'expérimentation entre pastoralisme et protection de la nature, Natures Sciences Sociétés, 20, 3, 310-317.

Jacobson S.K., Duff M.D., 1998. Training idiot savants: the lack of human dimensions in conservation biology, Conservation Biology, 12, 2, 263-267.

Mascia M.B., Brosius J.P., Dobson T.A., Forbes B.C., Horowitz L., McKean M.A., Turner N.J., 2003. Conservation and the social sciences, Conservation Biology, 17, 3, 649-650.

Muir M.J., Schwartz M.W., 2009. Academic research training for a nonacademic workplace: a case study of graduate student alumni who work in conservation, Conservation Biology, 23, 6, 1357-1368.

Noss R.F., 1997. The failure of universities to produce conservation biologists, Conservation Biology, 11, 6, 12671269.

Orr D.W., 1999. Education, careers, and callings: the practice of conservation biology, Conservation Biology, 13, 6, 1242-1245.

Reyers B., Roux D.J., Cowling R.M., Ginsburg A.E., Nel J.L., O'Farrell P., 2010. Conservation planning as a transdisciplinary process, Conservation Biology, 24, 4, 957-965.

Sandberg J., Tsoukas H., 2011. Grasping the logic of practice: theorizing through practical rationality, Academy of Management Review, 36, 2, 338-360.

Schwartz Y., 2007. Un bref aperçu de l'histoire culturelle $\mathrm{du}$ concept d'activité, activités, 4, 2, 122-133, http:// www.activites.org/v4n2/schwartz-FR.pdf.

Schwartz Y., 2010. Connaître et étudier le travail, Ergologia, 3, 93-104.

Star S.L., Strauss A., 1999. Layers of silence, arenas of voice: the ecology of visible and invisible work, Computer Supported Cooperative Work, 8, 1-2, 9-30.

Teel T.L., Manfredo M.J., 2010. Understanding the diversity of public interests in wildlife conservation, Conservation Biology, 24, 1, 128-139. 